\title{
$D$ region meteoric smoke and neutral temperature retrieval using the poker flat incoherent scatter radar
}

\author{
J. T. Fentzke, ${ }^{1,2}$ V. Hsu, ${ }^{3}$ C. G. M. Brum, ${ }^{4}$ I. Strelnikova, ${ }^{5}$ M. Rapp, ${ }^{6}$ and M. Nicolls ${ }^{7}$ \\ Received 14 September 2012; revised 17 October 2012; accepted 17 October 2012; published 15 November 2012
}

[1] This brief note describes the first measurement of the microphysical properties and variability of meteoric smoke particles (MSPs) at high latitude using the Poker Flat ISR $\left(65.1^{\circ} \mathrm{N}, 147.5^{\circ} \mathrm{W}\right)$. We present a novel technique for determining height resolved daytime $\mathrm{D}$ region neutral temperatures, which takes into account the presence of charged dust. We discuss the temporal/spatial variability and the relation to meteoric input observed and MSP microphysical properties in the polar mesopause region. The derived nanometer sized MSPs are consistent with size profiles derived previously using radar/rocket techniques and we note that our results imply a lack of heavy cluster ions below $85 \mathrm{~km}$ during the observing period. This provides a template for potential use at many other radar sites for the determination of microphysical properties of MSPs and day-time neutral temperature in the D region that show good general agreement with model and satellite temperature data during the observing period. Citation: Fentzke, J. T., V. Hsu, C. G. M. Brum, I. Strelnikova, M. Rapp, and M. Nicolls (2012), D region meteoric smoke and neutral temperature retrieval using the poker flat incoherent scatter radar, Geophys. Res. Lett., 39, L21102, doi:10.1029/2012GL053841.

\section{Introduction}

[2] Meteoric smoke particles (MSPs) result from the ablation and subsequent condensation of material from micrometeoroids that continually enter the Earth's atmosphere [Hunten et al., 1980]. Models predict that the characteristics (e.g., particle density and radii) of these nanometer sized MSPs vary as a function of latitude and altitude [Megner et al., 2008; Bardeen et al., 2008; Fentzke et al., 2009]. For example, predicted concentrations are on the order of $10^{2}-$ $10^{4} \mathrm{~cm}^{-3}$ in the D region (approximately between $70-90 \mathrm{~km}$ ) [Hunten et al., 1980]. MSPs are thought to play an important role in ice layer-related phenomena such as: Polar Mesospheric Clouds (PMCs) [Turco et al., 1982], Polar Mesospheric Summer Echoes (PMSEs) [Rapp and Lübken, 2004], as well USA.

${ }^{1}$ CoRA Division, NorthWest Research Associates, Boulder, Colorado,

${ }^{2}$ Space Department, Johns Hopkins University Applied Physics Laboratory, Laurel, Maryland, USA.

${ }^{3}$ Aerospace Engineering Sciences, University of Colorado at Boulder, Boulder, Colorado, USA.

${ }^{4}$ Arecibo Observatory, Arecibo, Puerto Rico.

${ }^{5}$ Leibniz Institute of Atmospheric Physics, Kühlungsborn, Germany.

${ }^{6}$ Deutsches Zentrum für Luft- und Raumfahrt Oberpfaffenhofen, Wessling, Germany.

${ }^{7}$ SRI International, Menlo Park, California, USA.

Corresponding author: J. T. Fentzke, Space Department, Johns Hopkins University Applied Physics Laboratory, 11100 Johns Hopkins Rd., Laurel, MD 20723-6099, USA. (jonathan.fentzke@jhuapl.edu)

(C)2012. American Geophysical Union. All Rights Reserved. 0094-8276/12/2012GL053841 as, atomic chemistry of mesospheric metals [Plane, 2011], condensation nuclei in Polar Stratospheric Clouds [Voigt et al., 2005], and transportation of mesospheric metals to the Earth's surface [Gabrielli et al., 2004].

[3] The microphyiscal properties and variability of MSPs are thought to contribute to the observed variability of PMCs [Gumbel and Megner, 2009]. These PMCs in turn change the albedo of the Earth as well as the chemistry of the upper atmosphere and may be a tracer for the impacts of global climate variability [Russell et al., 2009]. Recent rocket campaigns [e.g., Rapp et al., 2007], laboratory experiments [Saunders and Plane, 2011], incoherent scatter radar (ISR) measurements [Strelnikova et al., 2007; Fentzke et al., 2009] and more recently satellite observations from the The Aeronomy of Ice in the Mesosphere (AIM) mission [Hervig et al., 2012] have all reported the presence of dust, nominally between $65-95 \mathrm{~km}$.

[4] Here we present new results that investigate for the first time MSP properties derived from $\mathrm{D}$ region observations taken at the Poker Flat Incoherent Scatter Radar (PFISR) during 8 hours of the highest $\mathrm{D}$ region signal-to-noise (SNR) ratio from August 4, 2010. Previous work has demonstrated that PFISR is capable of observing D region phenomena such as PMSEs [Janches et al., 2009; Nicolls et al., 2009] and winds down to $60 \mathrm{~km}$ [Nicolls et al., 2010]. However, this is the first investigation of the temporal and spatial variability of charged dust in the mesopause region at high latitude using high power large aperture (HPLA) radar, which extends the discovery of MSP signatures at high latitude using the European Incoherent Scatter Scientific Association (EISCAT) UHF radar [Rapp et al., 2007]. The observing period reported here occurred right after the historical prolonged solar minimum (ascendant phase of the 24 solar cycle), and in a period of large geomagnetic fluctuation. The empirical methodology employed for this study is based on the theory developed by Cho et al. [1998] and relies on fitting the calculated autocorrelation function (ACF) of the measured $\mathrm{D}$ region spectra to determine particle radii under the assumption of mono-disperse particles (i.e., a single representative particle size at each altitude as opposed to a distribution) following the previous work of Strelnikova et al. [2007]. In addition, by modifying the fitting technique detailed in Strelnikova et al. [2007] and Fentzke et al. [2009] we demonstrate that it is possible to infer the height resolved neutral temperature in the mesopause region. In the following sections we present further details on the experimental setup, processing techniques and results from this effort that yield new information on MSP variability and neutral temperature in the polar mesopause region.

\section{Experimental Setup and Observations}

[5] Our experiment utilized the 128-panel Poker Flat ISR $\left(65.1^{\circ} \mathrm{N}, 147.5^{\circ} \mathrm{W}\right)$ operating at $449.3 \mathrm{MHz}$ in a 4 beam 
configuration using a 13-baud barker code, $5 \mu$ s sampling, and $2 \mathrm{~ms}$ IPP with a transmitted power of $\sim 1.72 \mathrm{MW}$. The observational period began at local midnight and continued until early afternoon ending at approximately 20 UT $(\mathrm{LT}+9)$, with appreciable D region signal between 12-20 UT. The returns were sampled between 40 and $140 \mathrm{~km}$ with approximately $750 \mathrm{~m}$ resolution. This investigation uses only the vertically pointed beam to reduce distortion with 256 pulses integrated to create spectra and autocorrelations (ACFs) with a frequency and time resolution of $3.9 \mathrm{~Hz}$ and $2 \mathrm{~ms}$, respectively. The data was median filtered to remove outliers [Nicolls et al., 2010] and Doppler corrected to within the spectral resolution of the experiment to remove the influence of noise sources and ensure that artificial spectral broadening during the integration period did not influence the derived sizes [Raizada et al., 2008; Fentzke et al., 2009]. Data products were created to match previous observations using HPLA radar [Strelnikova et al., 2007; Fentzke et al., 2009] by integrating without range binning to produce data products with a time resolution of $1 \mathrm{hr}$ and an altitude resolution of $750 \mathrm{~m}$ from approximately $70 \mathrm{~km}$ to $95 \mathrm{~km}$ where the $\mathrm{D}$ region signal was visible throughout the observing period from 12-20 UT. We note that sub-hour variability is not thought to be large due to the sedimentation rates and meteoric ablation [Megner et al., 2008; Fentzke et al., 2009; Rapp et al., 2010]. In addition, we ensure that the presence of PMSE does not influence our interpretation of the MSPs by removing any such events, noting that during the observing period between 12-20 UT, no PMSE was observed.

\section{Analysis}

[6] The theory regarding the incoherent scatter (IS) spectral shape of the D region in the presence of charged MSPs was developed by the pioneering work of Cho et al. [1998]. In the absence of charged MSPs and negative ions, IS theory predicts a broad Lorentzian spectral lineshape resulting from highly-damped ion-acoustic waves [Tepley and Mathews, 1978]. The spectral width of the Lorentzian $\left(\omega_{0}\right)$ is given by

$$
\omega_{0}=\frac{1}{\pi \tau_{0}}=\frac{32 \pi k_{B}}{\lambda_{r}^{2}} \frac{T}{m_{i} v_{i n}}
$$

Where $\lambda_{r}$ is the radar wavelength, $k_{B}$ is Boltzmann's constant, $v_{i n}$ is the ion-neutral momentum transfer collision frequency, $m_{i}$ is the ion mass, and the atmospheric temperature (T) is assumed to be equal for neutrals, ions, electrons, and particles. For $v_{i n}$, we adopt the ion-neutral momentum transfer collision frequency described by Tepley and Mathews [1978] assuming that the dominant ions are $\mathrm{NO}^{+}$and $\mathrm{O}_{2}^{+}$[Narcisi et al., 1972] leading to an $m_{i}$ of $31 \mathrm{amu}$.

[7] The presence of MSPs in the D region modifies the Lorentzian lineshape [Cho et al., 1998]. Strelnikova et al. [2007] showed empirically that the full expression presented in Cho et al. [1998] can be approximated as the sum of two Lorentzian spectra - or equivalently, the autocorrelation functions (which according to the Wiener-Khinchin theorem is given by the Fourier transform of the latter) can be approximated as the sum of two exponential decays under the assumption of mono-disperse particles:

$$
A C F(t)=A_{0} \cdot \exp \left(-t / \tau_{0}\right)+A_{1} \cdot \exp \left(-t / \tau_{1}\right)
$$

Where the decay times $(\tau)$ are related to the spectral width [Strelnikova et al., 2007] and the amplitudes (A) are proportional of returned power. The reader is referred to Cho et al. [1998] and Strelnikova et al. [2007] for further details. Applying the theory assuming mono-disperse particles with an ACF in the form of equation (2), independent of electron density and charge, one can show that the expression for the MSP size $\left(r_{\text {eff }}\right)$ takes the form:

$$
r_{e f f}=\frac{k_{b}}{2} \sqrt{\frac{3 \tau_{1}}{N_{n}} \sqrt{\frac{k_{b} T_{n}}{2 \pi M_{n}}}}-r_{n}
$$

Where $N_{n}$ is the neutral number density per meter cubed and $T_{n}$ is the neutral temperature in degrees Kelvin. The assumed mean mass of the neutral air molecule is $M_{n}=4.8 \times 10^{-26} \mathrm{~kg}$ and the mean radius of the neutral is $r_{n}=0.15 \mathrm{~nm}$. For the analysis, we adopt this empirical formulation, for the vertically pointed radar beam, which results in an ACF with a magnitude approximated by the sum of two exponential decays in the form of equation (2).

[8] The 1 hour integrated ACFs are used to derive the particle radii of MSPs in the D region, while the spectra are utilized to visually inspect the quality of the data. Due to noise in the data at lower altitudes and Voigt-like spectral profiles at higher altitudes in the D region, we limit our study to the altitude range of approximately $70-90 \mathrm{~km}$. From $12-$ 14 UT, the spectra at several altitudes are contaminated with noise spikes, which results in poor fits at various altitudes during the first few hours of our investigation. However, D region spectra are observed for the rest of the experiment, especially between $\sim 80-89 \mathrm{~km}$. The integrated ACFs are fitted to the summed exponentials given by equation (2) in the same manner as described by Strelnikova et al. [2007] and Fentzke et al. [2009]. This study also expands on the aforementioned fitting method by increasing the degrees of freedom in the fitting routine to investigate the feasibility of determining $T_{n}$ in addition to smoke radii, thus eliminating the dependence of the NRL-MSISE-00 model temperature on derived radii. This method expands on an initial study by Raizada et al. [2008], who used an isothermal barometric approximation to derive $T_{n}$ from $\mathrm{D}$ region spectra at Arecibo.

[9] Extracting MSP sizes from the ACFs requires solving equation (2) for the decay time in the exponential terms. From previous fitting methods [Strelnikova et al., 2007], the decay time $\tau_{0}$ and coefficient $A_{0}$ are determined using NRLMSISE-00 and the 0-lag to obtain the smoke-free background signal (D region without MSPs) from equation (1). Next the residual ACF is calculated by subtracting the background ACF from the integrated ACF, which results in the MSP ACF (D region MSP signal and noise). These residuals are then fitted for the remaining free parameters: $\tau_{1}$ and $A_{1}$. Particle sizes are then found by substituting the fitted $\tau_{1}$ along with the other background parameters into equation (3).

[10] The second fitting method fits the four free parameters in equation (2) simultaneously, as opposed to calculating the background and fitting the MSP ACFs separately. By solving the system of equations described by equations (1) and (3) with the fitted values it is possible to derive $\mathrm{T}_{n}$ without model temperatures, which provides the opportunity to uncover the temporally and spatially dynamic behavior as opposed to the climatological average nature of $\mathrm{T}_{n}$ in the mesopause region. 


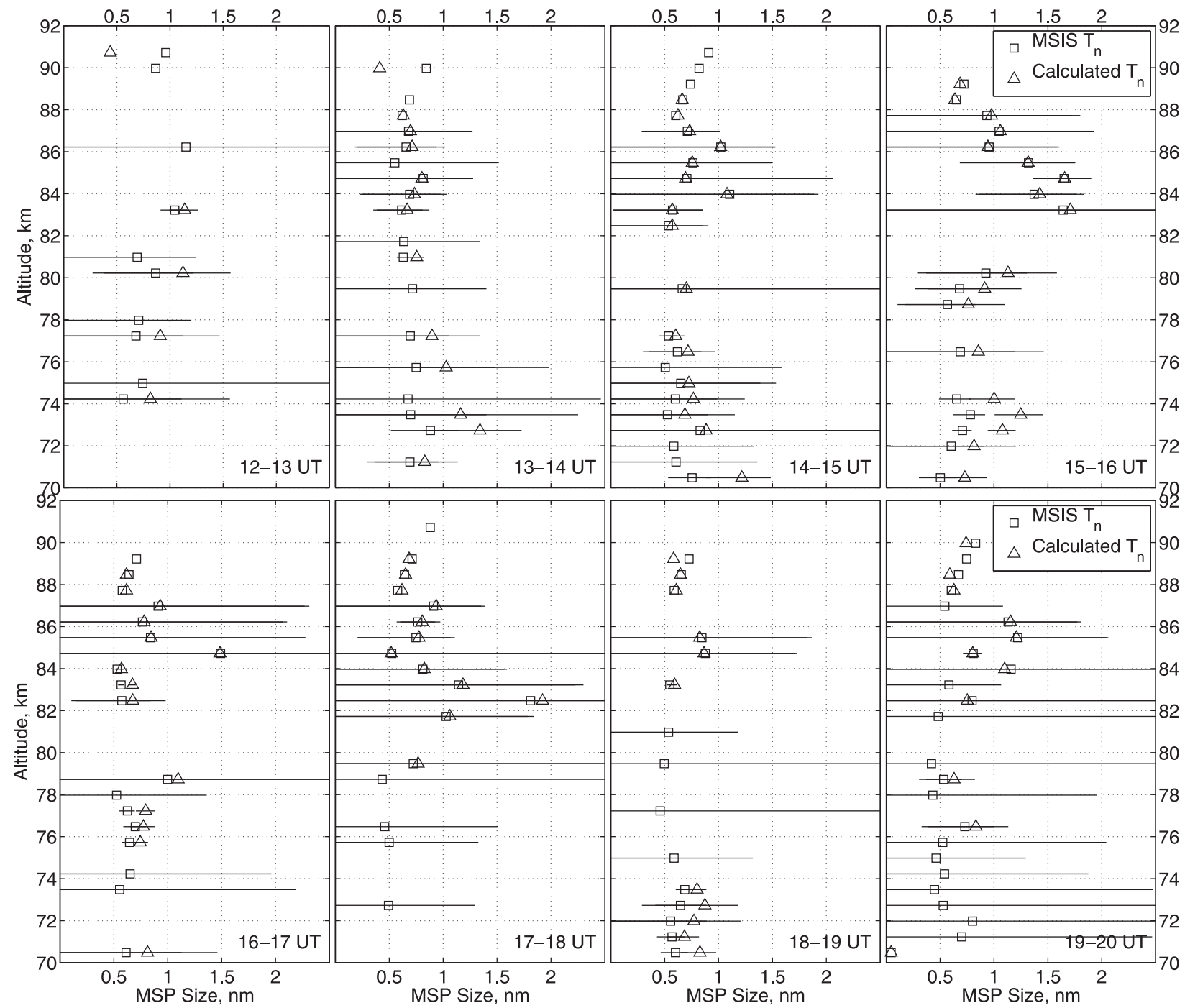

Figure 1. Time evolution of particle radii for an 8 hour observing period from 12-20 UT on August 4, 2010 with one sigma errors on the MSP decay time fit parameters. Two fitting methods are compared, one fitting the ACF residuals with two free parameters (squares), and the other fitting the complete ACF measurement with four free parameters (triangles).

[11] For both fitting methods, we fit the ACFs with the number of time lags that approach the stochastic noise floor where the real part of the ACF crosses zero [Mathews, 1986]. Both fitting methods assume a triangle weighting based on the de-correlation time of the underlying physical processes and show that neither the background nor the MSP component of the ACF dominate the fit [Strelnikova et al., 2007; Fentzke et al., 2009].

\section{Results and Discussion}

[12] Derived MSP sizes from both fitting methods are presented in Figure 1. To prevent incorrect fits from distorting the altitude profiles of MSP radii, we omit results from altitudes where bad fits (i.e., fits resulting in negative particle radius) occurred. The error bars on the MSP sizes were determined by using equation (3) with the 1-sigma upper and lower bounds of $\tau_{1}$, which represents the interquartile range of values. For the nominal operating mode and beam shape of PFISR of $1^{\circ}-1.5^{\circ}$ [see Nicolls et al., 2010] the potential error induced from beam broadening and shear [see Engler et al., 2005; Nastrom, 1997] is approximately $1 \mathrm{~Hz}$ based on background parameters from Nicolls et al. [2010] and assumptions from Fentzke et al. [2009]. In addition, Nicolls et al. [2010] showed spectral perturbations that were essentially zero below $75 \mathrm{~km}$.

[13] The variation in altitude and time for derived radii range from approximately $0.5 \mathrm{~nm}$ to $1.6 \mathrm{~nm}$. Figure 1 shows that MSP sizes are more variable than observations at lower latitude on the time scale of hours [Fentzke et al., 2009]. But further investigation is required to determine if this lack of variability can be attributed to the notion that much of the ablated meteoric material diffuses quickly into the background atmosphere without condensing, leaving a subset of remaining materials to coagulate into larger particles on the time scale of days or longer. However, in several altitude intervals the sizes are seen to double over the course of 1 to 2 hours $(\sim 85 \mathrm{~km})$. This is likely due to direct meteor ablation, but further analysis in the future on the high latitude chemistry, dynamics, and meteor input is required to confirm this conjecture. The radii at PFISR observed during this period 


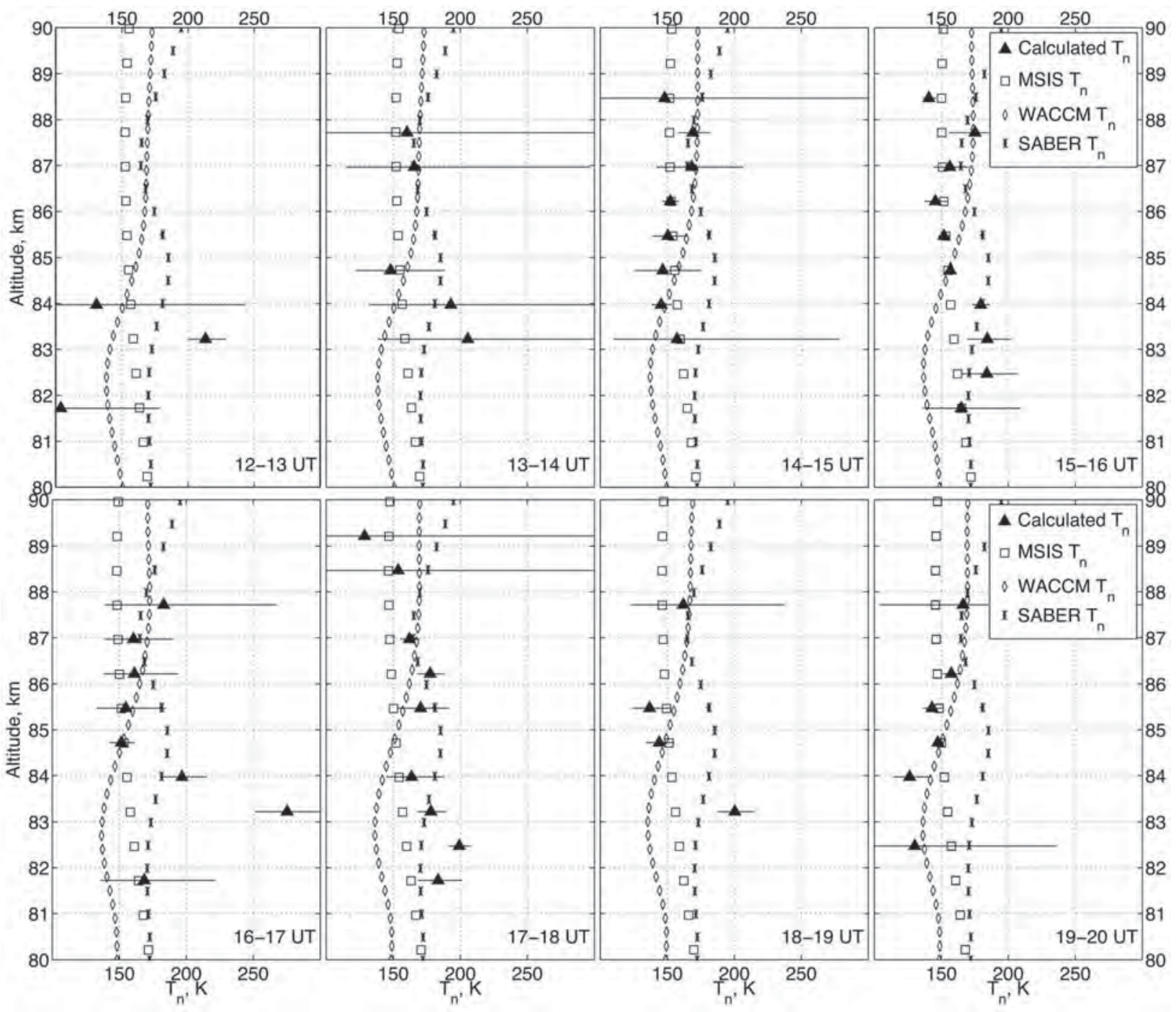

Figure 2. Time evolution of derived neutral temperatures (triangles) for 12-20 UT on August 4, 2010 with one sigma errors on the MSP decay time fit parameters. Measured SABER temperatures (asterisks), NRL-MSISE-00 model temperatures (squares), and WACCM temperatures (diamonds) are included for comparison. Both model and measurements from different sources show significant variability as a function of altitude owing to the complex dynamics of the $\mathrm{D}$ region.

from approximately $85-90 \mathrm{~km}$ were smaller by approximately $0.5 \mathrm{~nm}$ than previous results observed at Arecibo. Overall the derived particle radii are comparable to previous observational studies from rocket studies [Rapp et al., 2010] as well as chemistry modeling efforts [Hunten et al., 1980; Plane, 2011], satellite [Hervig et al., 2012] and global circulation models [Bardeen et al., 2008; Megner et al., 2008]. Thus providing further validation of derived MSP sizes in the D-region. The smaller radii at high latitude suggest that meridional circulation pulls fresh material away quickly resulting in a relatively constant distribution of MSP size with altitude. However, further study is needed to determine if this poleward circulation creates the observed bite outs or if charging and microphysical processes are responsible. While the radii are derived independent of charge, it is feasible that charging plays a role in the formation of bulk particles based on the chemical properties of the smoke constituents. This area is under active research [Saunders and Plane, 2011; Plane, 2011] and we hope to contribute to this effort in the future.
[14] Now, we describe results for a novel method of determining $T_{n}$ using the aforementioned theory under the assumption that the plasma is in thermal equilibrium with the surrounding neutral atmosphere due to the highly collisional regime [Fentzke et al., 2011] $\left(T_{e}=T_{i}=T_{n}\right.$, where $T_{e}$ and $T_{i}$ and $T_{n}$ are the electron, ion, and neutral temperatures, respectively). This assumption is valid for the highly collisional plasma in the $\mathrm{D}$ region under the conditions of our observation [Mathews, 1986]. By comparing the 2-parameter and the 4-parameter fitting methods in Figure 1, it is evident that there exists a small offset above $80 \mathrm{~km}$ and a consistent negative offset of about $0.1 \mathrm{~nm}$ for derived particle radii below $80 \mathrm{~km}$ when using the 2-parameter fit as opposed to the 4-parameter fit. However, above $80 \mathrm{~km}$, the two fitting methods agree quite well. Noting that the derived size is also influenced by the ion composition, neutral density and experimental setup. Figure 2 shows the neutral temperatures derived using the 4-parameter fit. The NRL-MSISE-00 model temperatures remain relatively constant throughout 
the altitude range under study; the model temperatures from NCAR-WACCM4 show a more dynamic temperature structure, but it does not capture the variability of measured SABERv1.07 temperatures averaged for 1 hour at $60^{\circ} \mathrm{N}$ from 12-13 UT; and the derived neutral temperatures over PFISR exhibit a more dynamic structure, especially at 14-16 UT. The model results, SABERv1.07 temperatures and this new technique all display variability and (dis)agreement depending on altitude and time. This highlights the variability in plausible temperatures, which are in reasonable agreement with the error bars of our measurements. The temperature profile suggests the influence of wave activity, but a study focusing on this aspect has not yet been carried out. We intend to compare this method in future work with daytime temperatures derived using lidar [Friedman, 2006] and satellite instrumentation [Mertens et al., 2001]. This method did not yield good agreement during the entire observing period, especially during periods of low photo-ionization. But, did yield reasonable altitude profiles above $80 \mathrm{~km}$ between 14 18 UT. Thus we believe the 4-parameter fitting method can provide a new approach for regularly determining $T_{n}$ during periods of heightened $\mathrm{D}$ region ionization (during sun-lit and storm periods) using HPLA-type radars.

\section{Conclusion}

[15] This observation has provided the first detection of meteor smoke size profiles at high latitude using PFISR. The resulting size profiles remain relatively unchanged at lower altitudes (below $85 \mathrm{~km}$ ) indicating a lack of cluster ions or an enhanced ion mass that appeared to modify the derived sizes in past observations [Fentzke et al., 2009]. The consistency of the derived sizes below $85 \mathrm{~km}$ may also indicate that the advection and sedimentation rate of larger particles is relatively constant over the duration of the observation resulting in a consistent measurement of the effective smoke radius as a function of altitude. The hourly variability in size of approximately 0.5 to $1.5 \mathrm{~nm}$ as a function of altitude is more variable than previously observed at Arecibo's equatorial latitude, the cause for this variability is as of yet unclear. However, we believe that there is evidence for production via meteor ablation during the period near sunrise [Fentzke and Janches, 2008] where the size triples during a 1 hour period from 15-16 UT; but, transport likely plays a major role in smoke evolution. Further investigation and multi-site measurements in conjunction with global models and neutral wind measurements are required to assess the relative contribution from transport versus local production.

[16] We have also presented a new technique for deriving neutral temperature using a 4-parameter fitting technique. The temperatures generally agree with other model and observational results, although the derived temperatures are more dynamic and variable. This is not surprising considering the climatological nature of models and observations that do not capture highly-variable small-scale temporal/spatial local dynamics. Noting that these observations were not originally intended to optimize the measurement of $T_{n}$. However, these results offer a promising new technique for range-resolved daytime neutral temperature, a quantity of great importance that has been provided only sparingly from several lidar sites and satellite observations.
[17] Acknowledgments. The PFISR is operated by SRI International under NSF ATM-0608577 cooperative agreement. J.T. Fentzke would like to recognize the time spent at the IAP on a visiting appointment as well as the thoughtful discussions with colleagues at Arecibo and SRI that made this work possible. The authors thank Charles Bardeen at NCAR for the WACCM results and Sam Yee at JHUAPL for the SABER data. These efforts were partially supported under NSF grant ATM-0924801 to NWRA, Inc.

[18] The Editor thanks the two anonymous reviewers for their assistance in evaluating this paper.

\section{References}

Bardeen, C. G., O. B. Toon, E. J. Jensen, D. R. Marsh, and V. L. Harvey (2008), Numerical simulations of the three-dimensional distribution of meteoric dust in the mesosphere and upper stratosphere, J. Geophys. Res., 113, D17202, doi:10.1029/2007JD009515.

Cho, J., M. Sulzer, and M. Kelley (1998), Meteoric dust effects on D-region incoherent scatter radar spectra, J. Atmos. Sol. Terr. Phys., 60(3), 349-357, doi:10.1016/S1364-6826(97)00111-9.

Engler, N., R. Latteck, B. Strelnikov, W. Singer, and M. Rapp (2005), Turbulent energy dissipation rates observed by Doppler MST Radar and by rocket-borne instruments during the MIDAS/MaCWAVE campaign 2002, Ann. Geophys., 23(4), 1147-1156.

Fentzke, J. T., and D. Janches (2008), A semi-empirical model of the contribution from sporadic meteoroid sources on the meteor input function in the MLT observed at Arecibo, J. Geophys. Res., 113, A03304, doi:10.1029/2007JA012531.

Fentzke, J. T., D. Janches, I. Strelnikova, and M. Rapp (2009), Meteoric smoke particle properties derived using dual-beam Arecibo UHF observations of D-region spectra during different seasons, J. Atmos. Sol. Terr. Phys., 71(17-18), 1982-1991, doi:10.1016/j.jastp.2009.09.002.

Fentzke, J. T., M. P. Sulzer, and S. A. González (2011), The effects of Bhatnagar-Gross-Krook, Brownian, and hard-sphere ion-neutral collision models on the incoherent scatter spectrum in the E region, J. Geophys. Res., 116, A01308, doi:10.1029/2010JA015340.

Friedman, J. S. (2006), The Arecibo potassium daylight receiver, in Proceedings of 23rd International Laser Radar Conference, edited by C. Nasagawa and N. Sugimoto, pp. 557-560, Conf. Steering Comm., Nara, Japan.

Gabrielli, P., et al. (2004), Meteoric smoke fallout over the Holocene epoch revealed by iridium and platinum in Greenland ice, Nature, 432, 1011-1014, doi:10.1038/nature03137.

Gumbel, J., and L. Megner (2009), Charged meteoric smoke as ice nuclei in the mesosphere: Part 1-A review of basic concepts, J. Atmos. Sol. Terr. Phys., 71, 1225-1235, doi:10.1016/j.jastp.2009.04.012.

Hervig, M. E., L. E. Deaver, C. G. Bardeen, J. M. Russell III, S. M. Bailey, and L. L. Gordley (2012), The content and composition of meteoric smoke in mesospheric ice particles from SOFIE observations, J. Atmos. Sol. Terr. Phys., 84-85, 1-6, doi:10.1016/j.jastp.2012.04.005.

Hunten, D. M., R. P. Turco, and O. B. Toon (1980), Smoke and dust particles of meteoric origin in the mesosphere and stratosphere, J. Atmos. Sci., $37,1342-1357$, doi:10.1175/1520-0469(1980)037<1342:SADPOM> 2.0.CO;2.

Janches, D., D. Fritts, M. Nicolls, and C. Heinselman (2009), Observations of D-region structure and atmospheric tides with PFISR during active aurora, J. Atmosp. Sol. Terr. Phys., 71(6-7), 688-696, doi:10.1016/j.jastp. 2008.08.015.

Mathews, J. (1986), Incoherent scatter radar probing of the $60-100-\mathrm{km}$ atmosphere and ionosphere, IEEE Trans. Geosci. Remote Sens., GE-24, 765-776, doi:10.1109/TGRS.1986.289625.

Megner, L., J. Gumbel, M. Rapp, and D. Siskind (2008), Reduced meteoric smoke particle density at the summer pole-Implications for mesospheric ice particle nucleation, Adv. Space Res., 41(1), 41-49, doi:10.1016/ j.asr.2007.09.006.

Mertens, C. J., M. G. Mlynczak, M. López-Puertas, P. P. Wintersteiner, R. H. Picard, J. R. Winick, L. L. Gordley, and J. M. Russell III (2001), Retrieval of mesospheric and lower thermospheric kinetic temperature from measurements of $\mathrm{CO}_{2} 15 \mu \mathrm{m}$ Earth Limb Emission under non-LTE conditions, Geophys. Res. Lett., 28(7), 1391-1394, doi:10.1029/ 2000GL012189.

Narcisi, R. S., A. D. Bailey, L. E. Wlodyka, and C. R. Philbrick (1972), Ion composition measurements in the lower ionosphere during the November 1966 and March 1970 solar eclipses, J. Atmos. Terr. Phys., 34(4), 647-658, doi:10.1016/0021-9169(72)90152-3.

Nastrom, G. D. (1997), Doppler radar spectral width broadening due to beamwidth and wind shear, Ann. Geophys., 15(6), 786-796, doi:10.1007/ s00585-997-0786-7. 
Nicolls, M., M. Kelley, R. Varney, and C. Heinselman (2009), Spectral observations of polar mesospheric summer echoes at $33 \mathrm{~cm}(450 \mathrm{MHz})$ with the poker flat incoherent scatter radar, J. Atmos. Sol. Terr. Phys., 71(6), 662-674, doi:10.1016/j.jastp.2008.04.019.

Nicolls, M. J., R. H. Varney, S. L. Vadas, P. A. Stamus, C. J. Heinselman R. B. Cosgrove, and M. C. Kelley (2010), Influence of an inertia-gravity wave on mesospheric dynamics: A case study with the Poker Flat Incoherent Scatter Radar, J. Geophys. Res., 115, D00N02, doi:10.1029/ 2010JD014042.

Plane, J. M. C. (2011), On the role of metal silicate molecules as ice nuclei, J. Atmos. Sol. Terr. Phys., 73(14-15), 2192-2200, doi:10.1016/ j.jastp.2010.07.008.

Raizada, S., M. P. Sulzer, C. A. Tepley, S. A. Gonzalez, and M. J. Nicolls (2008), Inferring D region parameters using improved incoherent scatte radar techniques at Arecibo, J. Geophys. Res., 113, A12302, doi:10.1029 2007JA012882.

Rapp, M., and F. Lübken (2004), Polar mesosphere summer echoes (PMSE): Review of observations and current understanding, Atmos. Chem. Phys, 4, 2601-2633, doi:10.5194/acpd-4-4777-2004.

Rapp, M., I. Strelnikova, and J. Gumbel (2007), Meteoric smoke particles: Evidence from rocket and radar techniques, Adv. Space Res., 40, 809-817, doi:10.1016/j.asr.2006.11.021.
Rapp, M., et al. (2010), Rocket-borne in situ measurements of meteor smoke: Charging properties and implications for seasonal variation, J. Geophys. Res., 115, D00I16, doi:10.1029/2009JD012725.

Russell, J. M., et al. (2009), The aeronomy of ice in the mesosphere (aim) mission: Overview and early science results, J. Atmos. Sol. Terr. Phys., 71(3-4), 289-299, doi:10.1016/j.jastp.2008.08.011.

Saunders, R. W and J. M. C. Plane (2011), A photo-chemical method for the production of olivine nanoparticles as cosmic dust analogues, Icarus, 212(1), 373-382, doi:10.1016/j.icarus.2010.12.019.

Strelnikova, I., M. Rapp, S. Raizada, and M. Sulzer (2007), Meteor smoke particle properties derived from Arecibo incoherent scatter radar observations, Geophys. Res. Lett., 34, L15815, doi:10.1029/2007GL030635.

Tepley, C. A., and J. D. Mathews (1978), Preliminary measurements of ion-neutral collision frequencies and mean temperatures in the Arecibo 80- to 100-km altitude region, J. Geophys. Res., 83(A7), 3299-3302, doi:10.1029/JA083iA07p03299.

Turco, R. P., O. B. Toon, R. C. Whitten, R. G. Keesee, and D. Hollenbach (1982), Noctilucent clouds: Simulation studies of their genesis, properties and global influences, Planet. Space Sci., 30(11), 1147-1181, doi:10.1016/ 0032-0633(82)90126-X.

Voigt, C., et al. (2005), Nitric acid trihydrate (NAT) formation at low NAT supersaturation in polar stratospheric clouds (PSCs), Atmos. Chem. Phys., 5(5), 1371-1380, doi:10.5194/acp-5-1371-2005. 